\title{
Factorization of Operators in Krein Spaces and Linear-Fractional Relations of Operator Balls
}

\author{
Victor Anatoly Khatskevich ${ }^{1}$, Valery Anatoly Senderov ${ }^{2}$ \\ ${ }^{1}$ Department of Mathematics, ORT Braude Academic College, Karmiel, Israel \\ ${ }^{2}$ Pyatnitskoe Highway, 23-2-156, Moscow, Russia \\ Email: victor_kh@hotmail.com, senderov@mccme.ru
}

Received July 22, 2012; revised September 18, 2012; accepted September 29, 2012

\begin{abstract}
We consider plus-operators in Krein spaces and generated operator linear fractional relations of the following form:

$$
\{F(K)=Q: A+B K=Q(C+D K) K \text { and } Q \text { are contractions between Hilbert space } X \text { and } Y\} \text {. }
$$

We study some special type of factorization for plus-operators $T$, among them the following one: $T=B U$, where $B$ is a lower triangular plus-operator, $U$ is a $J$-unitary operator. We apply the above factorization to the study of basical properties of relations (1), in particular, convexity and compactness of their images with respect to the weak operator topology. Obtained results we apply to the known Koenigs embedding problem, the Krein-Phillips problem of existing of invariant semidefinite subspaces for some families of plus-operators and to some other fields.
\end{abstract}

Keywords: Krein Space; Linear Fractional Relation; Plus-Operator; Factorization

\section{Introduction}

During World War II, one of the burning problems was the firing accuracy of the new rocket weapon called "Katyusha".

In 1943, S. L. Sobolev showed how to control the firing accuracy by using an eigenvector with a special eigenvalue of a linear operator $A$ that is self-adjoint with respect to an indefinite metric and whose canonical expansion contains a single negative square (Sobolev's paper was published in an administrative military journal and appeared in the public press only in 1960 [1]). In 1944, L. S. Pontryagin [2] generalized Sobolev's result as follows: he proved that, for an operator $A$ that is selfadjoint with respect to an indefinite metric and has a finite number $\kappa$ of negative squares, there exists a $\kappa$ dimensional invariant subspace such that the spectrum of the restriction to this subspace satisfies a special condition.

In 1950, M. G. Krein successfully used a fixed point principle to prove the equivalence between the existence of a specific invariant subspace of an operator $A$ and the fact that a linear-fractional mapping $F_{A}$ has a fixed point; he also obtained several new results in this field.

In 1959-1960, R. S. Phillips published several papers [3-5], where he showed how to solve some systems of differential equations by using extensions of invariant subspaces of several operator families.
Since that time the invariant subspace problem has become one of the central problems in the theory of spaces with indefinite metric (see [6]), and the spaces of the class axiomatized by M. G. Krein have been called Krein spaces.

Factorizations of different types are considered for operators in Krein spaces. The fundamental paper [7] deals with the so-called "J-polar decomposition of operators" which is an analog and a natural generalization of the usual polar decomposition of operators in Hilbert spaces.

Another type of decompositions is the factorization of operators of the form

$$
A=U B
$$

or

$$
A=C U^{\prime},
$$

where $B$ is an upper triangular operator, $C$ is a lower block triangular operator, and $U$ and $U^{\prime}$ are $J$-unitary operators.

It follows from the results presented below that each strict plus-operator (the definition is given below) admits factorization (1). We show that this is not the case for factorization (2).

Example. Let $\mathfrak{H}_{1}=C \operatorname{Lin}\left\{e_{i}, i=2, \cdots\right\}, \mathfrak{H}_{2}=\operatorname{Lin}\left\{e_{1}\right\}$, and let $A e_{i}=e_{i+1}$ for $i \in \dot{N}$. We also assume that $A=$ $B U$ is factorization (2), $a=U^{-1} e_{1} \neq 0$. Then

$B U a=B e_{1} \in \mathfrak{H}_{2}$. On the other hand, $A \mathfrak{H}=\mathfrak{H}_{1}$. There- 
fore, we have $A a=0$ and $a=0$.

(By Lin we denote the linear span of a set, and by $C$ Lin, its closure).

Factorizations (1) and (2) are rather useful tools for studying both the operators in spaces with indefinite metric and the so-called linear-fractional relations of operator balls. Several results contained in the present paper have not yet been published; the others were published recently and are practically unknown. But, to make our presentation clear and consistent, we begin with one of the classical schemes.

Let $\mathcal{K}$ be the open unit ball of the space $\mathcal{L}\left(\mathfrak{H}_{1}, \mathfrak{H}_{2}\right)$, where $\mathfrak{H}_{1}$ and $\mathfrak{H}_{2}$ are Hilbert spaces, and let $\mathcal{F}_{A}$ be a linear-fractional mapping of the form

$$
\mathcal{F}_{A}(K)=\left(A_{21}+A_{22} K\right)\left(A_{11}+A_{12} K\right)^{-1},
$$

where $A_{i j} \in \mathcal{L}\left(\mathfrak{H}_{1}, \mathfrak{H}_{i}\right), \quad i, j=1,2, \quad A=\left\|A_{i j}\right\|_{i, j=1}^{2}$, and $K \in \mathcal{K}$, and $A_{11}+A_{12} K$ is invertible.

Since the mappings satisfy the "chain rule" $\mathcal{F}_{A_{1} A_{2}}=\mathcal{F}_{A_{1}} \circ \mathcal{F}_{A_{2}}$, factorizations (1) and (2) lead to the decomposition of the linear-fractional mapping $\mathcal{F}_{A}$ into an automorphism $\mathcal{F}_{U}$ of the ball and a linear-fractional mapping $\mathcal{F}_{B}$ fixing the origin or into an affine linearfractional mapping $\mathcal{F}_{C}$ and an automorphism $\mathcal{F}_{U}$.

These facts have interesting applications. As an example, we mention the well-known problem, namely, the problem of the geometric and topological properties of some operator sets (see, for example, [8-14]).

The further development of this theory led to the problem of describing the structure of operator sets also in the case where $\mathcal{F}_{A}$ is a multivalued generating function.

Definition. The formula

$$
A_{21}+A_{22} K=K^{\prime}\left(A_{11}+A_{12} K\right),
$$

where $K, \quad K^{\prime} \in \mathcal{K}$, and $A_{i j} \in \mathcal{L}\left(\mathfrak{H}_{j}, \mathfrak{H}_{i}\right)$ for $i, j=1,2$, determines a linear-fractional relation (1.f.r.)

$\mathcal{F}=\mathcal{F}_{A}=\left\{K, K^{\prime}\right\}$ in $\mathcal{K}$. Note that if $A_{11}+A_{12} K$ is invertible for all $K \in \mathcal{K}$, then $\mathcal{F}_{A}$ becomes a linear fractional mapping defined earlier.

There does not exist any direct generalization of the mapping factorization theory to the case of linear-fractional relations. The point is that, even for very simple generating operators, the basic ("chain") equality may not hold in the case of relations.

Example. Let $\mathfrak{H}_{1}=C \operatorname{Lin}\left\{e_{i}\right\}$, where $i \in \mathbb{N},\left\{e_{i}\right\}$ is an orthonormal basis; $s e_{i}=e_{i+1} ; \quad u=\left(\begin{array}{cc}I & 0 \\ 0 & 0\end{array}\right)$ and $v=\left(\begin{array}{ll}s & 0 \\ 0 & 0\end{array}\right)$.

Clearly, $\mathcal{F}_{u v}$ are operators $K$ such that $K e_{1} \neq 0$. On the other hand, we have $\operatorname{Im} \mathcal{F}_{u}=\{0\}$.
But a significant generalization of the factorization theory (to arbitrary linear operators) opens new ways for studying the relations. Let us consider several results.

The most of them were obtained for bistrict plus-operators in [15].

Let us introduce the definitions and notation used in the present paper.

For more details, see [6].

\section{Basic Results}

Let $\mathfrak{H}$ be a Krein space:

$$
\mathfrak{H}=\mathfrak{H}_{1} \oplus \mathfrak{H}_{2},
$$

where $\mathfrak{H}_{1}$ and $\mathfrak{H}_{2}$ are nontrivial complex Hilbert spaces; $\mathcal{P}_{1}$ and $\mathcal{P}_{2}$ are the orthogonal projection operators corresponding to this decomposition of $\mathfrak{H}: \mathfrak{H}_{i}=\mathcal{P}_{i} \mathfrak{H}$, where $i=1,2$ and $\mathcal{P}_{1}+\mathcal{P}_{2}=I$. For any $x \in \mathfrak{H}$, we set $x_{i}=\mathcal{P}_{i} x$, where $i=1,2$; the indefinite metric $[\cdot, \cdot]$ in the space $\mathfrak{H}$ is given by the formula

$$
[x, y]=\left(x_{1}, y_{1}\right)-\left(x_{2}, y_{2}\right) \text {. }
$$

Here $x=x_{1}+x_{2}, \quad y=y_{1}+y_{2} \in \mathfrak{H}$, and $(\cdot, \cdot)$ is the (usual) inner product.

A set $S$ is said to be nondegenerate if $S \cap S^{[\perp]}=\{0\}$, where $S^{[\perp]}=\{y \in \mathfrak{H}:[y, z]=0$ for all $z \in S\}$.

By $\mathfrak{P}_{+}$and $\mathfrak{P}_{-}$we denote the sets of all nonnegative and all nonpositive vectors, respectively:

$$
\begin{aligned}
& \mathfrak{P}_{+}=\{x \in \mathfrak{H}:[x, x] \geq 0\}, \\
& \mathfrak{P}_{-}=\{x \in \mathfrak{H}:[x, x] \leq 0\} .
\end{aligned}
$$

To each lineal (linear manifold) $\mathcal{L}: \mathcal{L} \subset \mathfrak{P}_{+}$there corresponds a contraction $K(\mathcal{L}): \mathcal{P}_{1} \mathcal{L} \rightarrow \mathfrak{H}_{2}$ so that

$$
\mathcal{L}=\left\{x_{1}+K(\mathcal{P}) x_{1}, x_{1} \in \mathcal{P}_{1} \mathcal{L}\right\} .
$$

The operator $K(\mathcal{L})$ is called the angular operator of the lineal $\mathcal{L}$.

A lineal $\mathcal{L} \subset \mathfrak{P}_{+}$is said to be uniformly positive if

$$
[x, x] \geq d(\mathcal{L})\|x\|^{2}
$$

for all $x \in \mathcal{L}$ and some $d(\mathcal{L})>0$. This is equivalent to the condition $\|K(\mathcal{L})\|<1$. The uniformly negative lineal is defined similarly.

If the lineal $\mathcal{L}: \mathcal{L} \subset \mathfrak{P}_{+}$is maximal (with respect to inclusion), then $\mathcal{P}_{1} \mathcal{L}=\mathfrak{H}_{1}$, and hence $K(\mathcal{L}) \in \overline{\mathcal{K}}$. From now on, $\overline{\mathcal{K}}$ is the closed unit ball of the space $\mathcal{L}\left(\mathfrak{H}_{1}, \mathfrak{H}_{2}\right)$ of bounded linear operators acting from $\mathfrak{H}_{1}$ into $\mathfrak{H}_{2}$ and $\mathcal{K}$ is its interior: $K \in \mathcal{K} \Leftrightarrow\|K\|<1$.

A linear operator $A$ with domain $\mathfrak{D}(A) \subseteq \mathfrak{H}$ and range (image) $\operatorname{Im} A \subseteq \mathfrak{H}$ is called a plus-operator if

$$
A\left(\mathfrak{P}_{+} \cap \mathfrak{D}(A)\right) \subseteq \mathfrak{P}_{+} .
$$

The main objects of study in the present paper are the 
plus-operators $A: A \in \mathcal{L}(\mathfrak{H})$, where $\mathcal{L}(\mathfrak{H})$ denotes the set of all bounded linear operators with $\mathfrak{D}(A) \equiv \mathfrak{H}$.

A plus-operator $A$ is said to be strict if

$$
\mu(A)=\inf _{[x, x]=1}[A x, A x]>0,
$$

and bistrict if, along with $A$, the operator $A^{c}$ that is the adjoint of $A$ with respect to the indefinite metric $[\cdot, \cdot]$ : $[A x, y]=\left[x, A^{c} y\right], x, y \in \mathfrak{H}$ is also strict. In this case, $A^{c}=J A^{*} \bar{J}$, where $J=\mathcal{P}_{1}-\mathcal{P}_{2}$.

If $A$ is a bistrict operator, then $\mu(A)=\mu\left(A^{c}\right)$.

An operator $V \in \mathcal{L}(\mathfrak{H})$ is said to be $J$-expansive if

$$
[V x, V x] \geq[x, x] \text { for all } x \in \mathfrak{H},
$$

and $J$-bi-expansive if both operators $V$ and $V^{c}$ are $J$ expansive.

Each strict plus-operator is collinear to a $J$-expansive operator, and each bistrict plus-operator is collinear to a $J$-bi-expansive operator.

Each plus-operator $A$ determines a linear-fractional relation $\mathcal{F}_{A}$ on the ball $\overline{\mathcal{K}}$ :

$$
\mathcal{F}_{A}(K)=\left\{K^{\prime} \in \overline{\mathcal{K}}: A_{21}+A_{22} K=K^{\prime}\left(A_{11}+A_{12} K\right)\right\} .
$$

An operator $V$ is said to be focusing if there is a constant $\gamma(V)>0$ such that

$$
[V x, V x] \geq \gamma(V)\|V x\|^{2} \text { for all } x \in \mathfrak{P}_{+} .
$$

An operator $U \in \mathcal{L}(\mathfrak{H})$ is said to be J-unitary if $\operatorname{Im} U=\mathfrak{H}$ and

$$
[U x, U x]=[x, x] \text { for all } x \in \mathfrak{H} .
$$

Each $J$-unitary operator $V$ is determined by the following three parameters: $\Gamma \in \mathcal{K}$ and operators $V_{1}$ and $V_{2}$ unitary in $\mathfrak{H}_{1}$ and $\mathfrak{H}_{2}$, respectively, by the relation

$$
V=\left(\begin{array}{cc}
\left(I-\Gamma^{*} \Gamma\right)^{-\frac{1}{2}} V_{1} & \Gamma^{*}\left(I-\Gamma \Gamma^{*}\right)^{-\frac{1}{2}} V_{2} \\
\Gamma\left(I-\Gamma^{*} \Gamma\right)^{-\frac{1}{2}} V_{1} & \left(I-\Gamma \Gamma^{*}\right)^{\frac{1}{2}} V_{2}
\end{array}\right) .
$$

Theorem 1. Let $A \in \mathcal{L}(\mathfrak{H})$. Then the following assertions are equivalent:

1) $A_{11} A_{11}^{*}-A_{12} A_{12}^{*} \geq d A_{11} A_{11}^{*}$, where $d=d(A)>0$;

2) The lineal $A^{*} \mathfrak{H}_{1}$ is uniformly positive;

3) $A_{12}=A_{11} K^{*}$ for some $K \in \mathcal{K}$;

4) $A$ admits the factorization

$$
A=B U
$$

where $U$ is J-unitary and $B_{12}=0$.

Now we outline the proof of Theorem 1 .

$1) \Rightarrow 2$ ) Let $y=A^{*} x_{1}$, where $x_{1} \in \mathfrak{H}_{1}$. Then $y=\mathfrak{P}_{+}$, which implies

$$
[y, y] \geq d\left(A_{11}^{*} x_{1}, A_{11}^{*} x_{1}\right) \geq d \cdot\left(\frac{1}{2}(y, y)\right) .
$$

$2) \Rightarrow 3$ ) It suffices to consider the angular operator of the lineal $\mathcal{L}=A^{*} \mathfrak{H}_{1}$ and continue it to the entire space $\mathfrak{H}_{1}$ preserving the norm.

$3) \Rightarrow 4$ ) Let us consider the operator $V \in \mathcal{L}(\mathfrak{H})$ whose block-matrix in the basis $\left\{\mathfrak{H}_{1}, \mathfrak{H}_{2}\right\}$ has the form

$$
V=\left(\begin{array}{cc}
\left(I-K^{*} K\right)^{-\frac{1}{2}} & -K^{*}\left(I-K K^{*}\right)^{-\frac{1}{2}} \\
-K\left(I-K^{*} K\right)^{-\frac{1}{2}} & \left(I-K K^{*}\right)^{-\frac{1}{2}}
\end{array}\right) .
$$

$K$ is strictly contractive, as follows from (c). The operator $V$ (and hence the operator $V^{-1}$ ) is $J$-unitary (straightforward calculated). We set $B=A V$ and obtain $B_{12}=0, A=B U$, where $U=V^{-1}$.

4) $\Rightarrow$ 1) We have $A^{*}=U^{*} B^{*}$, where $B^{*} \mathfrak{H}_{1} \subseteq \mathfrak{H}_{1}$ and the $J$-unitary operator $U^{*}$ takes uniformly positive lineals to uniformly positive ones, which implies that the lineal $A^{*} \mathfrak{H}_{1}$ is uniformly positive, and hence assertion 1) follows.

It is natural to compare the statements of Theorem 1 with the following proposition.

Theorem 2. The plus-operator $A$ is exactly bistrict if $0 \in \rho\left(A_{11}\right)$, that is, 0 is a regular point of $A_{11}$, $\left\|A_{11}^{-1} A_{12}\right\|<1$, and $\left\|A_{21} A_{11}^{-1}\right\|<1$.

Lemma. Assume that the lineal $A \mathfrak{H}$ is nonnegative under the conditions of Theorem 2. Then A is a focusing strict plus-operator.

Proof of the lemma. It is easy to show that

$$
A=\left(\begin{array}{cc}
A_{11} & A_{11} Z \\
W A_{11} & W A_{11} Z
\end{array}\right),
$$

where $\|W\|<1$ and $\|Z\|<1$ (cf. [16], Proposition 2.3). Thus, for $x \in \mathfrak{P}$, we have

$$
\begin{aligned}
{[A x, A x] } & \geq\left(1-\|W\|^{2}\right) \cdot\left\|A_{11} x_{+}+A_{11} Z x_{-}\right\|^{2} \\
& \geq c_{1} \cdot\left\|A_{11}^{-1}\right\|^{-2}\left(\left\|x_{+}\right\|-\|Z\| \cdot\left\|x_{-}\right\|\right)^{2} \\
& \geq c_{2} \cdot\left(\left\|x_{+}\right\|-\|Z\| \cdot\left\|x_{+}\right\|\right)^{2} \\
& \geq c_{2} \cdot\left(1-\|Z\|^{2}\right) \cdot\left\|x_{+}\right\|^{2} \geq c_{3} \cdot\left(\frac{1}{2}\|x\|^{2}\right),
\end{aligned}
$$

where $c_{1}>0, c_{2}>0$ and $c_{3}>0$.

On the other hand, for some $A \in \mathcal{L}(\mathfrak{H})$, the inequality $[A x, A x] \geq c(A)\|x\|^{2}$, where $c(A)>0$, exactly holds for all $x \in \mathfrak{P}_{+}$if $A$ is a focusing strict plus operator. This can be easily proved using ([6], Theorem 2.4.11).

Proof of Theorem 2. It follows from the lemma and ([6], Corollary 2.4.5) that $A$ is a strict plus-operator. Since $0 \in \rho\left(A_{11}\right)$, this implies that the plus-operator $A$ is bistrict ([6], Theorem 2.4.17).

We illustrate the new methods and approaches listed above by an obvious example.

Namely, we use the method of operator factorization in indefinite spaces that genetically originates from T. Ya. 
Azizov's work [15]. This method allows us to prove that, for any strict plus-operator $A$ satisfying the condition $D=A_{11} A_{11}^{*}-A_{12} A_{12}^{*}$ is a definite operator: $D \geq 0$ or $D \leq 0$, the set $\operatorname{Im} \mathcal{F}_{A}$ is convex and compact in the weak operator topology (w.o.t.).

To prove this, we need some auxiliary assertions concerning the case of a strict plus-operator $A$ with an arbitrary operator $D$.

Lemma 3. Let $A$ be a strict plus operator, and let $\mathcal{L}=A^{*} \mathfrak{H}_{1}$. Then $\mathcal{L}^{\perp}$ and $\mathcal{L}^{[\perp]}$ are uniformly negative subspaces.

Proof. Let $x \in \mathcal{L}^{\perp}$. Then $(x, \mathcal{L})=\left(A x, \mathfrak{H}_{1}\right)=\left(\mathcal{P}_{1} A x, \mathfrak{H}_{1}\right)=0$. Thus, $x \in \operatorname{Ker}\left(P_{1} A\right)$, where $P_{1} A$ is a strict plus operator. Because of ([6], Proposition 2.4.14), the subspace $\operatorname{Ker}\left(P_{1} A\right)$ is uniformly negative. The end of the proof follows from the relation $\mathcal{L}^{\perp]}=J \mathcal{L}^{\perp}\left(\right.$ where $\left.J=\mathcal{P}_{1}-\mathcal{P}_{2}\right)$.

Now we prove that, in the case of an arbitrary strict plus-operator $A$, the lineal $A^{*} \mathfrak{H}_{1}$ contains a "sufficiently large" positive lineal. If $\operatorname{dim} \mathfrak{H}_{1}<\infty$, then $A^{*}$ is a strict plus-operator, which implies that the subspace $A^{*} \mathfrak{H}_{1}$ is positive and $\operatorname{dim}\left(A^{*} \mathfrak{H}_{1}\right)=\operatorname{dim} \mathfrak{H}_{1}$. Now we assume that $\mathfrak{H}_{1}=\infty$.

We have the following theorem.

Theorem 4. Let $\operatorname{dim} \mathfrak{H}_{1}=\infty$, and let $A$ be a strict plus-operator. Then, there exists an infinite-dimensional positive lineal in $A^{*} \mathfrak{H}_{1}$.

To prove this theorem, it suffices to prove the following assertion.

Lemma. Assume that, under the conditions of Theorem $4, \mathcal{L}_{1}$ is some maximal (with respect to inclusion) positive lineal contained in $A^{*} \mathfrak{H}_{1}$. Then $\operatorname{dim} \mathcal{L}_{1}=\infty$.

Proof of the lemma. Let $\mathcal{L}=A^{*} \mathfrak{H}_{1}$, and let $\operatorname{dim} \mathcal{L}_{1}<\infty$. Then $\mathcal{L}=\mathcal{L}_{1}[\dot{+}] \mathcal{M}$, where $\mathcal{M} \subset \mathfrak{P}_{-}$. On the other hand, using Lemma 3 , it is easy to prove that

$\mathfrak{H}=\mathcal{L}_{1}[\dot{+}] \overline{\mathcal{M}}[\dot{+}] \mathcal{L}^{[\perp]}$. Indeed

$$
\left(\mathcal{L}^{[\perp]}\right)^{[\perp]}=\overline{\mathcal{L}}=\overline{\mathcal{L}_{1}}[\dot{+}] \overline{\mathcal{M}}=\mathcal{L}_{1}[\dot{+}] \overline{\mathcal{M}} .
$$

Hence $\mathcal{L}_{1}$ is the maximum positive subspace, and this is a contradiction.

Further, we shall need the following proposition, which can also be proved by using Lemma 3 .

Proposition. If $A$ is a strict plus-operator, then $\overline{\mathcal{L}}=\overline{A^{*} \mathfrak{H}_{1}}$ is nondegenerate; if $\overline{\mathcal{L}}=\mathcal{L}_{+}[\dot{+}] \mathcal{L}_{-}$, where $\mathcal{L}_{ \pm} \subset \mathfrak{P}_{ \pm}$, then $\mathcal{L}_{+}$and $\mathcal{L}_{-}$are uniformly positive and uniformly negative subspaces, respectively.

Proof. The nondegeneracy of $\overline{\mathcal{L}}$ follows from the fact that $\mathcal{L}^{[\perp]}$ is (uniformly) negative. Further, since $\left(\mathcal{L}^{[\perp]}[\dot{+}] \mathcal{L}_{-}\right)[\dot{+}]\left(\mathcal{L}_{+}\right)=\mathfrak{H}$, the definite lineals in parentheses are closed and uniformly definite.

Corollary. If there exists a finite-dimensional maximal negative lineal $\mathcal{M}$ in $\mathcal{L}=A^{*} \mathfrak{H}_{1}$, then the lineal $\mathcal{L}[-] \mathcal{M}$ is uniformly positive.
We return to the case of a strict plus-operator $A$ with a definite operator $D$. It follows from Theorem 4 that $D \geq 0$. Further, it follows from the proposition that the lineal $A^{*} \mathfrak{H}_{1}$ is uniformly positive.

Thus, to complete the proof, it suffices to prove the following assertion.

Theorem 5. Assume that a plus-operator A satisfies the conditions of Theorem 1. Then the set $\operatorname{Im} \mathcal{F}_{A}$ is convex and compact in the weak operator topology (w.o.t.) (see the definition in [17]).

Proof (cf. [18]). Since $\mathcal{F}_{U}$ is a linear-fractional mapping of the ball $\overline{\mathcal{K}}$ onto itself, we have

$$
\mathcal{F}_{A}=\mathcal{F}_{B} \circ \mathcal{F}_{U}
$$

see ([19], Proposition 4.20). Because of the affine relation $\mathcal{F}_{B}$ and the equality $\mathcal{F}_{U}(\overline{\mathcal{K}})=\overline{\mathcal{K}}$, this readily implies that the set $\operatorname{Im} \mathcal{F}_{A}$ is convex.

It remains to prove that the set $\mathcal{F}_{B}(\overline{\mathcal{K}})$ is compact in w.o.t.

Since the ball $\overline{\mathcal{K}}$ is a compact set in w.o.t., its image $\mathcal{K}_{1}$ is also a compact set under the continuous mapping $K_{+} \rightarrow B_{21}+B_{22} K_{+}$. Hence $\mathcal{K}_{1}$ is a closed subset of the space $\mathcal{L}\left(\mathfrak{H}_{1}, \mathfrak{H}_{2}\right)$. Therefore, the complete preimage $\mathcal{F}_{B}(\overline{\mathcal{K}})$ of the set $\mathcal{K}_{1}$ under the continuous mapping $K_{+}^{\prime} \rightarrow K_{+}^{\prime} B_{11}$ of the ball $\overline{\mathcal{K}}$ is also closed. Thus, since $\overline{\mathcal{K}}^{+}$is a compact set, $\mathcal{F}_{B}(\overline{\mathcal{K}})$ is also a compact set. The proof of the theorem is complete.

We consider another application, namely, we show how both the factorization of operators and the linearfractional relations can be used to prove the existence of invariant subspaces.

Theorem 6. (cf. [20]. 1) Under the conditions of Theorem $1, \operatorname{Im} A_{12} \subseteq \operatorname{Im} A_{11}$.

2) Under the conditions of Theorem 1, assume in addition that

$$
A_{12}\left(A_{22}-A_{21} A_{11}^{-1} A_{12}\right) \in \gamma_{\infty}
$$

that is, $A_{12}\left(A_{22}-A_{21} A_{11}^{-1} A_{12}\right)$ is a compact operator. Then there exists an $\mathcal{L}$ such that $\mathcal{L} \in \mathfrak{M}_{+}$and $A \mathcal{L} \subseteq \mathcal{L}$.

To prove this theorem, we need the following auxiliary lemmas.

Lemma 7. The norms generated by different decompositions of the form $\mathfrak{H}=\tilde{\mathfrak{H}}_{1}[\dot{+}] \tilde{\mathfrak{H}}_{2}$ are equivalent.

Proof. This lemma readily follows from the Banach theorem.

Lemma 8. ([6], Remark 3.2.4) For the existence of the decomposition $\mathfrak{H}=\tilde{\mathfrak{H}}_{1}[\dot{+}] \tilde{\mathfrak{H}}_{2}$, where the matrix of the operator $A$ has the property $\tilde{A}_{12} \in \gamma_{\infty}$, it is necessary and sufficient that there exist a uniform contraction $Q \in \mathcal{L}\left(\mathfrak{H}_{2}, \mathfrak{H}_{1}\right)$ such that

$$
B=Q A_{22}+Q A_{21} Q-A_{12}-A_{11} Q \in \gamma_{\infty} .
$$

Lemma 9. ([6], Proposition 3.3.4) A subspace $\mathcal{L} \in \mathfrak{M}_{+}$ with an angular operator $K$ is exactly invariant under the 
action of a strict plus-operator $A$ if $K \in \mathcal{F}_{A}(K)$.

Proof of Theorem 6. 1) It follows from Theorem 1 that the factorization (2) and hence the inclusion $\operatorname{Im} A_{12} \subseteq \operatorname{Im} A_{11}$ exist, and hence $A_{11}^{-1} A_{12} \in \mathcal{L}\left(\mathfrak{H}_{2}, \mathfrak{H}_{1}\right)$.

2) By setting $Q=-A_{11}^{-1} A_{12}$, we obtain

$$
B=Q\left(A_{22}+A_{21} Q\right)=-A_{11}^{-1} A_{12}\left(A_{22}-A_{21} A_{11}^{-1} A_{12}\right) \in \gamma_{\infty} .
$$

It follows from Lemma 7 that the operator $A$ is bounded in the norm generated by the decomposition in Lemma 8. This, as usual, permits considering the linearfractional relation $\mathcal{F}_{\tilde{A}}$, where $\tilde{A}$ is the matrix of the operator $A$ in the basis $\left\{\tilde{\mathfrak{H}}_{1}, \tilde{\mathfrak{H}}_{2}\right\}$. It easily follows from the inclusion $\tilde{A}_{12} \in \gamma_{\infty}$ that the linear-fractional relation $\mathcal{F}_{\tilde{A}}$ is a closed (multivalued) mapping. Thus, we are under the conditions of Glicksberg's theorem [6], which implies that $\mathcal{F}_{\tilde{A}}$ has at least one fixed point in the ball $\|X\|_{1} \leq 1$ (here $X \in \mathcal{L}\left(\tilde{\mathfrak{H}}_{1} \tilde{\mathfrak{H}}_{2}\right)$ and \|\|$_{1}$ is the norm generated by the new decomposition).

By Lemma 9, this implies that the operator $A$ has a maximal nonnegative invariant subspace.

\section{Conclusions}

We note that statement 2) of Theorem 6 holds for any operator with the property

$$
\operatorname{Im} A \subset \mathfrak{P}_{+} .
$$

Indeed, for any $\mathcal{L} \in \mathfrak{M}_{+}$such that $\operatorname{Im} A \subseteq \mathcal{L}$, we have $A \mathcal{L} \subseteq \mathcal{L}$.

In particular, each nonstrict plus-operator in a Krein space satisfies condition (3).

We note that the method of mapping factorization constantly finds new applications. In particular, in [18], precisely this method permits obtaining new conditions under which the given operator is a plus-operator.

\section{REFERENCES}

[1] S. L. Sobolev, "On the Motion of a Symmetric Top with a Cavity Filled with a Liquid," Zh. Prikl. Mekhan. i Tekhn. Fiz., No. 3, 1960, pp. 20-55.

[2] L. S. Pontryagin, "Hermitian Operators in Spaces with Indefinite Metric," Izvestiya Akademii Nauk SSSR, Seriya Matematicheskaya, Vol. 8, 1944, pp. 243-280.

[3] R. S. Phillips, "Dissipative Operators and Hyperbolic Systems of Partial Differential Equations," Transactions of the American Mathematical Society, Vol. 90, No. 2, 1959, pp. 193-254. doi:10.1090/S0002-9947-1959-0104919-1

[4] R. S. Phillips, "Dissipative Operators and Parabolic Partial Differential Equations," Communications on Pure and Applied Mathematics, Vol. 12, No. 2, 1959, pp. 249276. doi:10.1002/cpa.3160120206

[5] R. S. Phillips, "The Extensions of Dual Subspaces Invariant under an Algebra," Proceedings of the International Symposium on Linear Spaces, Jerusalem, 5-12 July
1960, pp. 366-398.

[6] T. Ya. Azizov and I. S. Iokhvidov, "Foundations of Theory of Linear Operators in Spaces with Indefinite Metric," Nauka, Moscow, 1986.

[7] M. G. Krein and Yu. L. Shmul'yan, "J-Polar Representation of Plus Operators," Materialy Issledovaniya, Vol. 1, No. 2, 1966, pp. 172-210.

[8] V. A. Khatskevich, "Some Global Properties of FractionalLinear Transformations," Operator Theory, Vol. 73, 1994, pp. 355-361.

[9] V. A. Khatskevich and V. S. Shulman, "Operator Fractional-Linear Transformations: Convexity and Compactness of Image, Application," Studia Mathematica, Vol. 116, No. 2, 1995, pp. 189-195.

[10] V. A. Khatskevich and L. Zelenko, "Indefinite Metrics and Dichotomy of Solutions for Linear Differential Equations in Hilbert Spaces," Chinese Journal of Mathematics, Vol. 24, No. 2, 1996, pp. 99-112.

[11] V. A. Khatskevich and L. Zelenko, "The Fractional-Linear Transformations of the Operator Ball and Dichotomy of Solutions to Evaluation Equations," Contemporary Mathematics, Vol. 204, 1997, pp. 149-154. doi:10.1090/conm/204/02628

[12] V. A. Khatskevich, "Generalized Fractional Linear Transformations: Convexity and Compactness of the Image and the Pre-Image; Applications," Studia Mathematica, Vol. 137, No. 2, 1999, pp. 169-175.

[13] V. A. Khatskevich and L. Zelenko, "Bistrict Plus-Operators in Krein Spaces and Dichotomous Behavior of Irreversible Dynamical Systems," Operator Theory: Advances and Applications, Vol. 118, 2000, pp. 191-203.

[14] V. Khatskevich and V. A. Senderov, "On Convexity, Compactness, and Non-Emptiness of Images and Preimages of Operator Linear-Fractional Relations," Doklady Akademii Nauk, Vol. 69, No. 3, 2004, pp. 409-411.

[15] T. Ya. Azizov, "On Extension of Invariant Dual Pairs," Ukrainian Mathematical Journal, Vol. 41, No. 7, 1989, pp. 958-961. doi:10.1007/BF01060700

[16] V. Khatskevich, V. Senderov and V. Shulman, “On Operator Matrices Generating Linear Fractional Maps of Operator Balls," Contemporary Mathematics, Vol. 364, 2004, pp. 93-102. doi:10.1090/conm/364/06679

[17] N. Dunford and J. Schwartz, "Linear Operators," Wiley, New York, 1958.

[18] V. Khatskevich and V. A. Senderov, "On Operator Sets Generated by Plus-Operators," Vestnik Voronezhskogo Gosudarstvennogo Universiteta, Seriya Fizika, Matematika, No. 2, 2010, pp. 170-174.

[19] V. Khatskevich, M. Ostrovskii and V. Shulman, "Linear Fractional Relations for Hilbert Space Operators," Mathematische Nachrichten, Vol. 279, No. 8, 2006, pp. 875890. doi:10.1002/mana.200310400

[20] T. Azizov and V. Khatskevich, "A Theorem on Existence of Invariant Subspaces for J-Bi-Expansive Operators," Operator Theory: Advances and Applications, Vol. 198, 2009, pp. 41-48. 